and secondary endpoints but an update of the trial with intermediate clinical results will be presented at the conference. Conclusion We provide early/interim results of the use of the River stent. This novel stent has been effective for the treatment of venous sinus stenosis without any short-term serious adverse events or device related complications.

Disclosures A. Patsalides: None.

\section{IMAGING TRIAGE OF LATE WINDOW PATIENTS WITH ACUTE ISCHEMIC STROKE. A COMPARATIVE STUDY USING MULTI-PHASE CT ANGIOGRAPHY VS CT PERFUSION}

${ }^{1} \mathrm{M}$ Almekhlafi*, ${ }^{2} \mathrm{~W}$ Kunz, ${ }^{3} \mathrm{R}$ McTaggart, ${ }^{4} \mathrm{M}$ Jayaraman, ${ }^{1} \mathrm{M}$ Najm, ${ }^{5} \mathrm{~S}$ Ahn, ${ }^{6} \mathrm{E}$ Fainardi, ${ }^{7} \mathrm{M}$ Rubiera, ${ }^{8} \mathrm{~A}$ Khaw, ${ }^{9} \mathrm{~A}$ Zini, ${ }^{10} \mathrm{M}$ Hill, ${ }^{10} \mathrm{~A}$ Demchuk, ${ }^{10} \mathrm{M}$ Goyal, ${ }^{10} \mathrm{~B}$ Menon. ${ }^{1}$ University of Calgary, Calgary, AB, Canada; ' $U$ niversity of Calgary, Munich, AB, Canada; ${ }^{3}$ Warren Alpert Medical School of Brown University, Providence, $R I_{;}{ }^{4}$ Warren Alpert Medical School of Brown University, Calgary, AB, Rl; ${ }^{5}$ Chosun University School of Medicine, Gwang Ju, Korea, Republic of; ${ }^{6}$ University Hospital, Ferrara, Ferrara, Italy; ${ }^{7}$ Hospital Vall d'Hebron, Barcelona, Spain; ${ }^{8}$ University of Western Ontario, London, ON, Canada; ${ }^{9}$ Maggiore Hospital, Bologna, Italy; ${ }^{10}$ University of Calgary, Calgary, AB, Canada

\subsection{6/neurintsurg-2019-SNIS.225}

Background Current guidelines recommend the use of perfusion imaging for selection of patients for endovascular thrombectomy (EVT) beyond six hours from onset. The role of collateral imaging in this time window is not established.

Methods We used data from a prospective multi-center observational study where all stroke patients with suspected large vessel occlusion underwent imaging with single- and multiphase CT angiography (mCTA) as well as CT perfusion. For this analysis, we only included patients presenting beyond six hours from onset/last known well time. Two blinded reviewers judged patients' eligibility for EVT using published collateral imaging (mCTA), compared to CT perfusion (using DAWN and DEFUSE-3 trials) selection criteria. All perfusion images were processed using an automated commercial software. The outcomes of patients eligible for EVT using mCTA, DAWN, or DEFUSE-3 criteria were compared using multivariable logistic regression modeling. Model predictive characteristics were assessed using c-statistic for the receiver operating curve, Akaike information criterion (AIC), and Bayesian information criterion (BIC).

Results Of 614 patients, 86 patients presented beyond six hours from onset/last known well (median 9.6 hours, IQR 4.1 hours). Median age was 71 years (IQR 14 years), 48.8\% were females, median baseline NIHSS was $12(\mathrm{IQR}=11)$. Thirty-five patients $(40.7 \%)$ received EVT of which good functional outcome (90 day modified Rankin scale 0-2) was achieved in $47 \%$. Collateral-based imaging paradigms significantly modified the treatment effect of EVT on clinical outcome i.e. 90-day mRS 0-2 (P interaction $=0.007)$. The mCTA-based regression model best fit the data for 90-day outcome (C statistic 0.86, 95\% CI 0.77 to 0.94 ) and was associated with least information loss (AIC 95.7, BIC 114.9) when compared to CTP based models. Perfusion imaging paradigm using DEFUSE-3 criteria had better predictive properties than the DAWN trial criteria.

Conclusion Collateral-based imaging paradigm using mCTA compares well with CTP in selecting patients for EVT in the late time window.

Disclosures M. Almekhlafi: None. W. Kunz: None. R. McTaggart: None. M. Jayaraman: None. M. Najm: None. S. Ahn:
None. E. Fainardi: None. M. Rubiera: None. A. Khaw: None. A. Zini: None. M. Hill: None. A. Demchuk: None. M. Goyal: None. B. Menon: None.

\section{BUILDING EFFECTIVE STROKE SYSTEMS OF CARE THROUGH A REGIONAL EMERGENCY MEDICAL SERVICES (EMS) COALITION AND DATA TRANSPARENCY INITIATIVE: THE SOUTH FLORIDA EXPERIENCE}

${ }^{1} \mathrm{~B}$ Mehta* ${ }^{2} \mathrm{P}$ Antewy, ${ }^{2} \mathrm{R}$ Katz ${ }^{3} \mathrm{~J}$ Sessa, ${ }^{4} \mathrm{~K}$ Scheppke, ${ }^{5} \mathrm{P}$ Pepe, ${ }^{6} \mathrm{R}$ Hanel, ${ }^{7} \mathrm{M} M$ ${ }^{8} \mathrm{C}$ Gutierrez, ${ }^{9} \mathrm{~J}$ Romano, ${ }^{10} \mathrm{R}$ Nogueira, ${ }^{11} \mathrm{~A}$ Jadhav, ${ }^{12} \mathrm{~T}$ Leslie-Mazwi, ${ }^{9} \mathrm{R}$ Sacco. ${ }^{1}$ Neurosurgery, Memorial Healthcare System, Hollywood, FL; ${ }^{2}$ Emergency Medicine, Memorial Healthcare System, Hollywood, FL; ${ }^{3}$ Neurology, Memorial Healthcare System, Hollywood, FL; ${ }^{4}$ Palm Beach County Fire Rescue, West Palm Beach, FL; ${ }^{5}$ Emergency Medicine, UT Southwestern Medical Center, Dallas, TX; ${ }^{6}$ Neurosurgery, Baptist Health, Jacksonville, FL; ${ }^{7}$ Neurosurgery, USF Health, Tampa, FL; ${ }^{8}$ Neurology, University of Miami, Miami, FL; ${ }^{9}$ Neurology, University of Miami School of Medicine, Miami, FL; ${ }^{10}$ Neurosurgery, Emory University School of Medicine, Atlanta, GA; ${ }^{11}$ Neurology, University of Pittsburgh Medical Center, Pittsburgh, PA; ${ }^{12}$ Neurosurgery, Massachusetts General Hospital, Boston, $M A$

10.1136/neurintsurg-2019-SNIS.226

Introduction Advances in acute stroke treatment of emergency large vessel occlusions (ELVO) have led to a transformation in the delivery of stroke care. Because of the importance of time to treatment with endovascular thrombectomy (EVT), there has been renewed emphasis on improving stroke systems of care. In south Florida, there has been a proliferation of stroke centers over the past decade claiming to offer EVT due to the practice of self-attestation by the Agency for Healthcare Administration (AHCA). Concurrently, there are over a dozen EMS agencies in the region, leading to variability in pre-hospital protocols.

Methods To address the fragmented stroke landscape, in 2015 local EMS Medical Directors along with NeuroInterventionalists (NI) developed the South Florida Stroke Coalition (SFSC), and collaborated with the University of Miami Florida Stroke Registry (UM FSR) team. The core tenets were to: 1) Develop pre-hospital EMS stroke protocols; 2) Optimize in-hospital stroke processes to reduce times to treatment for ELVO; 3) Encourage all tri-county stroke centers to submit data to AHA/ASA Get with the Guidelines-Stroke (GWTG-S) program and participate in the UM FSR; 4) Develop a sustainable data transparency model for CSCs to allow EMS Medical Directors to understand quality of stroke care in their region; 5) Eliminate the practice of AHCA self-attestation of CSCs and require stroke centers to apply for Joint Commission certification.

Results The SFSC and the UM FSR analyzed AHCA criteria for Comprehensive Stroke Center (CSC) designation and compared them to those of the Joint Commission to better understand limitations of self-attestation. A sustainable mechanism for local hospitals to share stroke data with the EMS Medical Directors in their catchment areas was created through a regional dashboard initiative (figure 1). The following key variables were selected by the SFSC to provide a better understanding of the quality of care: 1) treatment rates for tPA and EVT, 2) door-to-needle time for tPA, 3) door-to-puncture time for EVT, 4) outcomes at 90 days using modified Rankin scale. Conclusion To our knowledge, the Florida Stroke Registry Regional Dashboards developed as a grassroots effort between the SFSC and the UM FSR represents the first-of-its-kind, EMS-driven effort to improve stroke systems of care in a 